\title{
Atomic-Resolution Analysis of Perovskite from the Early Solar System
}

\author{
Thomas J. Zega ${ }^{1,2}$, Venkat Manga ${ }^{2}$, Ken Domanik ${ }^{1}$, and Krishna Muralidharan ${ }^{2}$. \\ 1. Lunar and Planetary Laboratory, University of Arizona, Tucson, AZ, U.SA. \\ 2. Department of Materials Science and Engineering, University of Arizona, Tucson, AZ.
}

Calcium- and aluminum-rich inclusions (CAIs) are $\mathrm{mm}$ - to $\mathrm{cm}$-sized objects that occur in primitive meteorites. They have radiometric age dates that exceed those of all other solar materials, and so are the oldest solids to have formed in the solar system [e.g., 1]. So named because of the Ca- and Al-rich materials they contain, CAIs are composed of mineral phases formed at very high temperatures and which are predicted by thermodynamic models to be among the first solids to have condensed within a cooling gas of solar composition [e.g., 2]. Their analysis can provide a glimpse into some of the earliest chemical and physical processes to have transpired during solar-system formation. Perovskite, nominally $\mathrm{CaTiO}_{3}$, is the major Ti-bearing phase in type-A CAIs and is predicted to host rare-earth elements and (minor) lithophile elements [3]. Here we report on the structure of several perovskite grains in a type-A CAI from the Allende CV3 chondrite down to the atomic level to gain insight into their origins.

A petrographic thin section of the Allende CV3 chondrite (TS25, U. Chicago) was examined using an FEI Helios focused-ion-beam scanning electron microscope (FIB-SEM), equipped with an EDAX energy-dispersive spectrometer (EDS), located at the Lunar and Planetary Laboratory, University of Arizona. We acquired backscattered electron images (BSE) and EDS spectrum images of a local part of the CAI. Selected perovskite grains were extracted using the FEI easylift micromanipulator on the Helios and thinned to electron transparency using previously described methods [4]. All samples were ion polished down to $5 \mathrm{keV}$ to remove the amorphous damage layer created by higher-voltage milling. The FIB cross sections were analyzed using the newly developed $200 \mathrm{keV}$ Hitachi HF5000 transmission electron microscope (TEM) located at Hitachi High Technologies (Mito, Japan). The HF5000 is equipped with a cold-field-emission gun, an aberration-corrector for scanning TEM (STEM) imaging, and a large solid angle Si-drift Oxford EDS system. It is capable of rapid (minutes) X-ray mapping of entire FIB sections and atomic-resolution imaging $(80 \mathrm{pm})$.

A localized region within the inclusion hosts abundant perovskite grains (Fig. 1). The grains are tens of $\mu \mathrm{m}$ in size and contain subhedral morphologies. Four perovskite grains were chosen for more detailed investigation using TEM. C straps were deposited along lines transecting the perovskite grains and their interfaces with the surrounding material.

Bright-field TEM imaging reveals that the FIB sections of the perovskite and surrounding material have varied microstructural complexity. All are polyphasic with grains that range in size from nm to several $\mu \mathrm{m}$. Perovskite from two of the FIB sections contains inclusions that EDS spectrum imaging reveals are spinel (nominally $\mathrm{MgAl}_{2} \mathrm{O}_{4}$ ). The inclusion from section ' $\mathrm{D}$ ' exhibits a lathic morphology, measures approximately $100 \times 150 \mathrm{~nm}$ in size, and occurs adjacent to a $\mathrm{Ca}-\mathrm{Mg}$-Al-silicate. The inclusion from section ' $C$ ' is significantly larger, measuring approximately $300 \times 400 \mathrm{~nm}$ in size. High-resolution imaging and selected area electron diffraction shows that it is twinned along the [111] direction. EDS spectrum imaging reveals that $\mathrm{V}$ is segregated to atomic columns located in a single atomic plane at the twin boundary (Fig. 2). 
Equilibrium thermodynamic calculations predict that both perovskite and spinel will condense from a gas of solar composition. Perovskite is predicted to condense at $1593 \mathrm{~K}$ and at $1441 \mathrm{~K}$, whereas spinel is predicted to condense at $1397 \mathrm{~K}$ [3]. The high-temperature phase of perovskite is cubic, whereas the lower temperature phase is orthorhombic [5]. The microstructure we observe here is inconsistent with these predictions, raising the question of whether equilibrium condensation occurred for this particular CAI.

We hypothesize that the solid solution of spinel (with dissolved V) exhibits a higher condensation temperature than the solid solution of perovskite. Based on the hypothesis, the spinel condensed first as a single crystal at a relatively higher condensation temperature than the perovskite. As temperature of the local system dropped to the condensation temperature of perovskite, the high-temperature cubic form condensed around the spinel. As temperature continued to decrease, the perovskite underwent a displacive phase transition from the cubic to orthorhombic form. This phase transition imparted shear stress to the spinel crystal, and it became twinned in response to the applied stress. Further, we hypothesize that the $\mathrm{V}$, which was originally homogeneously distributed in the spinel, segregated at the induced twin, driven by an energetically favorable diffusion, forming a plane of atomic columns parallel to the [111] direction. Preliminary DFT studies suggest that the presence of solute elements (e.g. V) shift the relative stability of the different solid phases and their equilibrium condensation temperatures. [6]

\section{References:}

[1] Amelin Y. et al. Science, 297, (2002) $1678-1683$.

[2] Ebel D. S. Met. and the Early S. Sys. II (2006), 253-277.

[3] Lodders K. (2003) Astrophys. J., 591, 1220-1247.

[4] Zega T. J. et al. Meteoritics \& Planet. Sci., 42 (2007), 1373-1386.

[5] Roushown, A. et al. J. of Solid State Chemistry, 178, (2005) 2867-2872.

[6] We thank Drs. Hiromi Inada and Keitaro Watanabe at Hitachi High Technologies (Mito, Japan) for their assistance with the HF5000 TEM. Research supported by NASA grant NNX15AJ22G (TJZ).

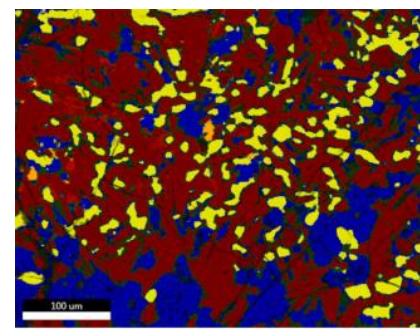

Fig. 1. SEM-EDS false-color spectrum image from a type-A CAI (Allende CV3 chondrite). Yellow = perovskite, red $=$ hibonite, blue $=$ melilite .

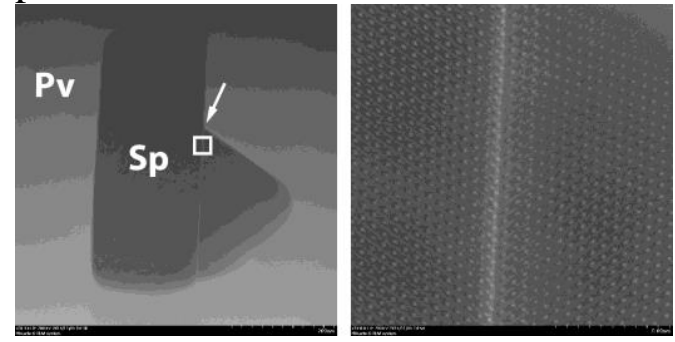

Fig. 2. STEM-HAADF images of spinel inclusion within perovskite. Overview image (left); atomicresolution image (right) of area outlined by white box in left-hand image. 